\title{
Création de valeur en contexte pénitentiaire : le Projet Artistique et Culturel du Musée du Louvre à la Maison Centrale de Poissy
}

Creating value in a penitentiary context: the artistic and cultural project of the Louvre Museum in the penitentiary at Poissy

Creación de Valor en Contexto de una Penitenciaría: El Proyecto Artístico y Cultural del Museo del Louvre en la Casa Central de Poissy

\section{Camille Jutant et Gaëlle Lesaffre}

\section{(2) OpenEdition}

\section{Journals}

Édition électronique

URL : http://journals.openedition.org/iss/428

DOI : $10.4000 /$ iss. 428

ISSN : 2306-4161

Éditeur

ICOM - International Council of Museums

Édition imprimée

Date de publication : 1 décembre 2015

Pagination : 135-150

ISSN : 2309-1290

Référence électronique

Camille Jutant et Gaëlle Lesaffre, «Création de valeur en contexte pénitentiaire : le Projet Artistique et Culturel du Musée du Louvre à la Maison Centrale de Poissy », ICOFOM Study Series [En ligne], 43b | 2015, mis en ligne le 06 février 2018, consulté le 22 avril 2019. URL : http:// journals.openedition.org/iss/428; DOI : 10.4000/iss.428 


\title{
Création de valeur en contexte pénitentiaire : le Projet Artistique et Culturel du Musée du Louvre à la Maison Centrale de Poissy
}

\author{
Camille Jutant \\ Université Lumière Lyon2, laboratoire ELICO (EA 4147) - \\ France \\ Gaëlle Lesaffre \\ Centre Norbert Elias (UMR Cnrs 8562) - France
}

\section{Introduction}

Depuis 2009, le musée du Louvre met en œuvre une programmation artistique et culturelle destinée aux personnes détenues en collaboration avec des centres pénitentiaires en Île-de-France. Si, en tant que monument, le musée du Louvre affirme par son existence le projet politique d'assigner un lieu à la Culture, par ce type de programmation, il déplace la mise en exposition des œuvres et des savoirs hors de son lieu et réaffirme le fait que le patrimoine culturel est un bien qui mérite d'être commun (Jeanneret, 2011). II associe à ces projets un dispositif d'étude et de recherche-action (Musée du Louvre-Service études et recherche 2010, 2013 ; Rostaing \& Touraut, 2012) pour comprendre quels sont les bénéfices, mais aussi les difficultés et les ambivalences, produits par ce type de projet en milieu carcéral. De cette façon, il participe à la fois à l'intervention culturelle en prison et à questionner la complexité de cette " intervention culturelle » (Soulier, 1985), déjà soulevée par d'autres études (Salle, 2011 ; Benguigui, 2011; Chauvenet, Rostaing \& Orlic, 2008 ; Chauvenet, 2006 ; Andrieu, 2011 ; Frize, 2004).

Le musée du Louvre n'est, ainsi, pas le seul à s'être engagé sur le terrain de l'action culturelle en prison. Comme le rappellent Rostaing et Touraut (2012), trois protocoles ont été signés depuis 1986 entre le ministère de la Justice et celui de la Culture afin d'affirmer leur détermination à garantir aux détenus un accès à la culture, et une centaine de partenariats entre des institutions culturelles et des établissements pénitentiaires ont été établis. Toutefois, la particularité de l'intervention culturelle réalisée par le musée du Louvre à travers deux projets mis en place à la maison centrale de Poissy tient à la valorisation par les acteurs du projet, contrairement à ce que Siganos (2008) indique avoir observé de manière générale dans le milieu carcéral, du moins jusqu'en 2007.

II est clair que le statut prestigieux du musée du Louvre joue un rôle dans la valorisation des projets qu'il propose par les personnes détenues, mais aussi par l'Administration pénitentiaire (AP), et notamment par les conseillers pénitentiaires d'insertion et de probation (CPIP) qui ne manquent pas de souligner le caractère exceptionnel du partenariat avec le "Louvre ». La proximité instaurée avec l'institution culturelle via son président directeur, alors Henri Loyrette, et l'expertise reconnue aux artistes intervenants, clairement identifiés en tant qu'artistes et non en tant qu'animateurs, influencent aussi la perception qui est celle de l'action culturelle initiée par le Louvre en partenariat avec l'Administration pénitentiaire. 
Le présent article souhaite comprendre quels sont les effets de ce projet en termes de valeur pour les participants, c'est-à-dire essentiellement pour les personnes détenues et non les personnels de I'AP ou les intervenants du projet. Pour cela, il propose d'interroger plus spécifiquement l'appropriation, par les personnes détenues, de ce projet, construit autour de l'installation de deux moulages de sculptures appartenant aux collections du musée et de la programmation de quatre ateliers de création (vidéo, danse, sculpture et création sonore) ainsi que d'un cycle de conférences menées par des conservateurs du musée et des jardins, des chercheurs, des universitaires, un restaurateur, etc. Cette analyse s'appuie sur les résultats de la dernière étude menée à l'occasion du projet organisé à la maison centrale de Poissy entre septembre 2012 et mai 2013, cet établissement pénitentiaire étant situé dans le centre de la vieille ville de Poissy, dans un ancien couvent de religieuses ursulines reconstruit plusieurs fois, et prenant en charge les personnes détenues pour de longues peines et les condamnés les plus « difficiles $»$.

Une approche qualitative a été privilégiée afin de pouvoir rendre compte, de façon compréhensive et en profondeur, des différentes étapes du projet, et notamment, de l'engagement des participants et des résistances des non-participants, du déroulement des ateliers et des conférences et enfin des bénéfices retirés de l'expérience. L'étude s'appuie sur 20 observations (conférences, séances d'ateliers, réunions collectives, journée de restitution) et 39 entretiens menés auprès de ces différents acteurs, entre septembre 2012 et juin 2013.

Cet article ne décrit pas les difficultés qui ont été rencontrées dans la conduite du projet, conséquences du poids du système de contraintes lié à l'univers carcéral, mais aussi d'une certaine défiance des personnes détenues vis-à-vis de l'administration pénitentiaire. Notons, néanmoins, que l'étude a permis de réitérer des résultats déjà manifestes dans les études précédentes menées par le musée du Louvre (op. cit.), concernant la tension entre l'accès à la culture et la satisfaction des besoins primaires dans le cadre de la vie en détention. Ajoutons que cet article ne présente pas non plus les nombreux arbitrages auxquels ont fait face les détenus au moment du choix de leur participation au projet, fruit d'une négociation de chacun avec son histoire personnelle, ses propres freins, motivations et attentes liés à la situation carcérale proprement dite ou au projet lui-même, et déterminants dans leur participation au projet du Louvre. La prise en compte de ces arbitrages a permis de comprendre la façon dont ils ont composé avec l'ensemble des facteurs liés à l'univers carcéral ou au projet lui-même, et dont ils ont construit des postures de réception, c'est-à-dire des attentes, des goûts et des motivations.

L'attention sera portée ici sur une autre dimension du projet: le répertoire de valeurs qui peut émerger d'un tel projet. L'article décrit rapidement le projet - des ateliers et des conférences - articulé

\footnotetext{
49 « La maison centrale de Poissy est située au centre historique de la vieille ville, à proximité de la Collégiale, dans un ancien couvent de religieuses ursulines fondé en $1645(. .$.$) / La prison est presque complètement reconstruite sous le Second Empire.$ En 1975, un nouveau bâtiment cellulaire est construit sur quatre niveaux (...). Une maison centrale reçoit les condamnés les plus difficiles. Leur régime de détention est essentiellement axé sur la sécurité. ॥ Extrait du site Internet du ministère de la Justice (dernière consultation le 22/01/2015) http://www.annuaires.justice.gouv.fr/etablissements-penitentiaires-10113/directioninterregionale-de-paris-10121/poissy-10648.html
} 
autour de l'exposition des deux moulages du musée du Louvre, avant de présenter les espaces de valeur individuelle cités par les personnes détenues interrogées et d'analyser, dans une quatrième et dernière partie, les espaces de valeur collective tels qu'ils ont été révélés par l'étude.

\section{Répertoires individuels et collectif de la valeur}

Par valeur, il faut entendre l'importance estimée par un jugement subjectif. Analysant comment se structurent et s'organisent des mondes de l'art, Howard Becker distingue bien jugement esthétique et valeur esthétique, le jugement esthétique étant un phénomène caractéristique d'une activité collective émis par ceux qui y participent, et « l'interaction de tous les participants [engendrant] un sentiment commun de la valeur de ce qu'ils produisent collectivement ॥, autrement dit engendrant de la valeur esthétique (Becker, 1988).

La valeur est ici considérée dans sa relation à la fois aux objets rencontrés par les détenus et aux expériences vécues, et qualifiées au cours des entretiens. En somme, il ne s'agira pas ici de distinguer la valeur comme propriété d'un objet (des sculptures en tant qu'œuvres par exemple) de la valeur comme propriété d'une expérience, mais plutôt de comprendre comment se tisse chez les détenus le sentiment qu'une valeur personnelle et qu'une valeur pour le groupe sont en jeu dans leur expérience des sculptures, des ateliers, des conférences, des rencontres avec les personnels du Louvre, avec les artistes intervenants, etc. On voit bien que la notion de valeur peut prendre ici de nombreuses tonalités et que la valeur esthétique n'est certainement pas la seule à opérer dans le répertoire des expériences des enquêtés. On peut imaginer ainsi que les valeurs d'authenticité, d'autonomie, de dépassement de soi, de moralité, d'originalité, de rareté, de responsabilité, de vérité, de virtuosité, de responsabilité, de sociabilité, etc. soient à l'œuvre dans les processus d'appropriation du projet par les personnes détenues.

L'effort d'explicitation, effectué par les personnes détenues qui ont accepté de faire part de leur expérience auprès des chercheuses, a permis de révéler qu'elles accordent véritablement de la valeur au projet et aux différents éléments qui le constituent. On peut distinguer trois grands registres de valeurs : esthétique et émotionnelle, liée au plaisir esthétique ou aux émotions ressenties, en termes de valeurs humaines défendues ou respectées, permises par les échanges et leur qualité, en terme de valeur professionnelle ou technique, issue des savoir-faire appris ou mobilisés. On retrouve ces trois registres de la valeur exprimée par les personnes détenues chez Siganos, qui les qualifie non pas de valeur mais d'« enjeux qui justifient l'opinion des acteurs sur une activité à laquelle ils ont participé » (Siganos, op. cit.), à savoir des enjeux esthétique, éthique et technique.

Le répertoire des espaces de valeurs que met au jour l'analyse de l'appropriation des activités par les détenus se déploie à l'échelle individuelle mais aussi, différemment, à l'échelle collective.

\section{Identification des espaces de valeur individuelle}

Deux espaces de valeur sont ici manifestes : celui de la relation avec les intervenants et celui de la démarche artistique. Dans le premier cas, la valeur est éthique et technique: elle est associée à la présence de personnes extérieures, au statut de ces personnes 
(intervenants et conférenciers) ainsi qu'à leurs savoir-faire et compétences et, enfin, aux échanges désintéressés que ces personnes proposent aux participants ; dans le deuxième cas, la valeur est plutôt émotionnelle : elle est liée à la démarche créative dans laquelle les personnes détenues participant aux ateliers sont invitées à entrer.

Pour certains détenus, on pourrait même considérer que ces espaces sont des espaces "intermédiaires " au sens que Donald Winnicott ${ }^{50}$ donne à ce terme (Lehmann, 2009). II s'agit des espaces dans lesquels l'individu investit son environnement par le jeu créatif, utilise des symboles et manie des objets qui le renvoient à sa capacité à agir sur le monde. Ici, la créativité caractérise une attitude face à la réalité extérieure qui consiste à prendre plaisir à ce que l'individu fait :

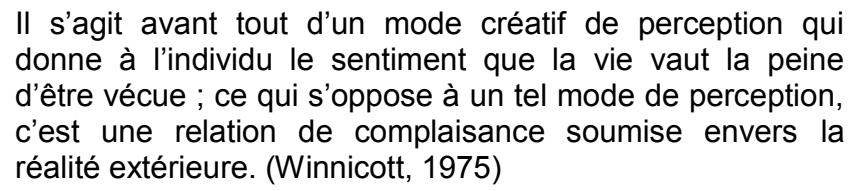
Identification des "espaces d'intérêt général " où la valeur
prend un sens collectif

Ces espaces sont le fruit de débats et d'échanges entre personnes détenues à l'occasion, d'une part, de l'introduction des sculptures dans la cour de la maison centrale et, d'autre part, des dynamiques collectives des ateliers de création. Ils engagent respectivement une forme de valeur éthique et une forme de valeur technique. Ces espaces sont aussi des espaces "d'intérêt général » : ils sont permis quand un consensus se forme, implicitement ou explicitement, au sein de l'ensemble de la population pénitentiaire autour du respect d'un objet ou du bon déroulement d'événements qui, finalement, vont profiter à tous. C'est ainsi que les moulages du Louvre ont été respectés, malgré les débats qu'ils ont pu susciter et que des moments de rencontre collective avec les personnes venues de l'extérieur, comme les conférences, les ateliers ou, plus encore, la restitution, se sont bien déroulés.

Le projet du Louvre n'est, bien sûr, pas le seul à pouvoir prétendre donner l'occasion de susciter la manifestation d'un "intérêt général ». La coordinatrice du projet au CPIP parle de ces espaces, qu'elle qualifie de "zones de non-problème ", de "lieux sacrés " auxquels on ne touche pas. Elle cite, par exemple, les parloirs ou les $U_{V F}{ }^{51}$ qui correspondent à des moments où la population pénale est incitée à prendre en compte l'extérieur lorsqu'il est valorisé et valorisant, et plus seulement centrée sur les rapports entre les personnes détenues et l'administration pénitentiaire.

\footnotetext{
${ }^{50}$ Cet espace est défini par Winnicott comme un « lieu de repos, pour l'individu engagé dans cette tâche humaine interminable qui consiste à maintenir à la fois séparées et reliées l'une et l'autre, réalité intérieure et réalité extérieure ", cité par Jean-Pierre Lehmann (2009).

${ }^{51}$ Unités de vie familiale. Les UVF sont des appartements de 2 ou 3 pièces dans lesquels la personne détenue (prévenue ou condamnée) peut recevoir sa famille et ses proches.
} 


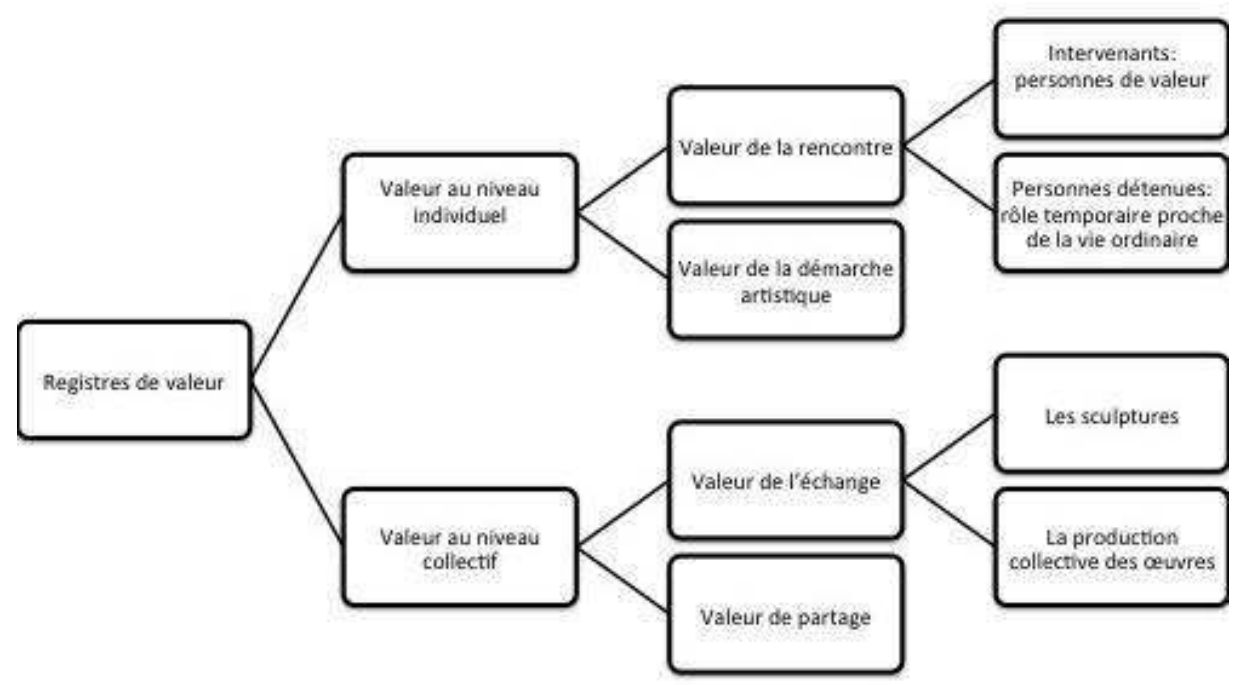

Figure $n^{\circ} 1$ : synthèse des registres de valeurs en jeu dans l'appropriation $d u$ projet par les personnes détenues

\section{Une exposition de moulages, pivot d'un programme d'ateliers et de conférences}

Le deuxième projet du Louvre à la maison centrale de Poissy s'est articulé autour d'une exposition de deux moulages de sculptures appartenant aux collections du musée du Louvre: le Gladiateur Borghèse (vers 100 avant J.-C.) et la Vénus Callipyge de François Barois $\left(\mathrm{XVII}{ }^{e}\right.$ siècle). Ces deux sculptures ont été exposées de juillet 2012 à juin 2013 au cœur même du quartier de détention, dans la cour dite "cour $n^{\circ} 5$ ", menant au bâtiment d'hébergement et à la régie industrielle des établissements pénitentiaires $\left(\operatorname{RIEP}^{52}\right)$, lieu de travail des personnes détenues. L'installation des deux sculptures a été l'occasion d'une collaboration étroite avec les équipes de paysagistes de l'atelier horticulture, engagé à la maison centrale depuis le début de l'année 2012: le choix de l'emplacement des moulages et la réalisation des socles ont été le fruit d'une réflexion commune (entre les équipes du musée du Louvre et les paysagistes) sur l'inscription de l'exposition dans le projet de réaménagement de la cour $n^{\circ} 5$.

Le projet proposait un cycle de dix conférences thématiques élaboré autour de ces deux sculptures et animées par des intervenants aux profils variés, conservateurs du Louvre, conférencier, restaurateur, historiens d'art. Mais le volet le plus important du projet destiné aux personnes détenues était constitué de quatre ateliers de création, c'est-à-dire des espaces d'expression, d'expérimentation, de création en lien avec les œuvres exposées et encadrés par des artistes, ciaprès nommés " intervenants " autour de différentes disciplines : la vidéo, la création sonore, la sculpture et la danse (cet atelier s'est terminé avant son terme, au bout de trois séances). Contrairement aux autres ateliers, l'atelier de création sonore s'est appuyé sur une

\footnotetext{
${ }^{52}$ La Régie Industrielle des Etablissement Pénitentiaire (RIEP) est gérée par le SEP, Service de l'Emploi Pénitentiaire, un service à compétence nationale du ministère de la Justice en charge, dans le cadre de sa mission de réinsertion, de développer des activités de travail et de formation au sein des établissements pénitentiaires. Le SEP RIEP regroupe 48 ateliers répartis dans 24 établissements offrant des savoir-faire dans une dizaine de métiers : travail du métal, du bois, du cuir, confection, imprimerie, reliure, informatique, traitement du son et de l'image, façonnage et agriculture.
} 
démarche préexistante à la RIEP au sein de l'atelier de numérisation sonore. II se distinguait également par le fait que chaque personne détenue y participant a travaillé sur son propre projet de création, alors que les deux autres ateliers ont systématiquement donné lieu à un travail collectif. En lien avec les deux moulages, l'atelier sculpture visait à poursuivre l'aménagement de la cour $n^{\circ} 5$; l'atelier danse visait à permettre un travail et une réflexion sur le corps et le mouvement. L'atelier vidéo visait, quant à lui, à poursuivre le travail initié lors du projet du Louvre à Poissy, autour de l'exposition de peintures. II faut noter que parmi les cinq intervenants, seulement deux d'entre eux étaient déjà intervenus - et régulièrement - en milieu carcéral.

La plus grande partie du projet du Louvre à Poissy a eu lieu dans les espaces réservés à la détention : la salle de peinture ${ }^{53}$ et le dojo, placés dans le bâtiment des activités, détournés de leur usage principal pour devenir atelier de sculpture ou salle de conférences et atelier de danse, la salle de l'activité vidéo, permanente, située au premier étage du bâtiment d'hébergement, la salle de création sonore prévue à cet effet au niveau de l'atelier de numérisation sonore de la RIEP, la cour 5 où ont été exposés la Vénus Callipyge et la Gladiateur Borghèse avant le début des ateliers ${ }^{54}$.

Les ateliers ont donné lieu, le 7 juin 2013, à une après-midi de présentation des créations réalisées dans les trois ateliers menés à leur terme, en présence des intervenants, d'une partie des personnes détenues participantes et non participantes, et de personnes invitées.

\section{L'émergence d'espaces de valeur individuelle}

Les activités proposées par le musée sont investies par les participants qui y recherchent ou y découvrent deux types d'espace de valeur: (1) l'espace de la rencontre avec des intervenants, métaphore du lien avec l'extérieur et la vie ordinaire et (2) l'espace de la démarche artistique engagée au cours des ateliers. Dans les deux cas, ces espaces de valeur ont une opérativité pour les personnes détenues d'un point de vue individuel. Ce sont leur propre rôle en tant que détenu, en tant que personne appartenant à la société, leur lien avec leur famille ou leur capacité créative qui sont en jeu.

\section{Les relations avec le monde du dehors et la mise à distance du monde carcéral}

Le premier espace de valeur est celui de la rencontre entre le détenu et de nouvelles personnes, de surcroît des personnes n'étant pas détenues, ne travaillant pas dans la maison centrale et permettant aux détenus de découvrir des réalités du " monde de dehors » qu'ils ne connaissent pas ou plus forcément.

La valeur des intervenants. Un premier type de valeur est en jeu dans la rencontre entre les personnes détenues et les intervenants extérieurs : ces derniers sont des objets de valeur. Dans le cas des conférences, c'est le savoir des intervenants qui est transmis et donc

\footnotetext{
${ }^{53}$ La mise à disposition de cet espace n'a pas été sans conséquence sur le déroulement de l'atelier sculpture mais aussi sur la conception des créations, puisque outils et matériaux devaient être contrôlés, transportés et remportés lors de chaque séance pour laisser la salle dans son état initial.

${ }^{54}$ Cette cour, permettant d'accéder à la RIEP, à l'UCSA (Unité de consultation et de soins ambulatoires) et au bâtiment d'hébergement, est traversée par l'ensemble des personnes détenues amenées à quitter leur cellule, par l'ensemble des personnels de I'UCSA et de la RIEP et par de nombreux surveillants.
} 
estimé par les auditeurs, alors que dans les ateliers les intervenants sont jugés sur leur professionnalisme et leurs compétences, et plus spécifiquement pour leur passion, c'est-à-dire leur enthousiasme et l'amour de leur métier, pour leur générosité ou encore pour leur expertise :

Je ressens ça de (l'intervenante de l'atelier vidéo), qu'elle a envie de le faire, qu'elle s'investit ! (Détenu participant 3$)^{55}$

Il est sculpteur lui (l'intervenant de l'atelier sculpture) ; il est artiste. Donc il s'y connaît quand même. (Détenu participant 7)

La valeur de la relation (ou la valeur du rôle). Un deuxième type de valeur est en jeu dans les ateliers et concerne cette fois les personnes détenues participantes. Les ateliers permettent d'exister autrement que dans un rôle stéréotypé de détenu, de donner de la valeur aux personnes qui s'investissent, notamment lorsque la relation entre détenu participant et intervenant laisse place à une relation de travail entre deux personnes, dans des rôles proches de ceux de la vie « ordinaire » :

- Toi en tant qu'individu tu es sorti du lot et tu sors de ce moule uniforme pour... pour retrouver une vraie conscience de toi.

- Et c'est souhaitable?

- C'est indispensable. On est mort sinon. (Détenu participant 2)

Elle (l'intervenante de l'atelier vidéo) comprenait les choses; elle faisait comme si on étaient des gens libres, des gens de dehors. (Détenu participant 9 participant projet Louvre 1)

Les détenus ne sont pas les seuls à mentionner le bénéfice de se sentir autre que détenu; les personnels de la prison le notent également :

Il y en a qui pensent que le fait d'être en détention euh... I/s sont plus rien pour la société. Ils sont là, ils ont qu'à croupir et puis c'est bon. Le fait qu'il y ait des choses comme ça où on pense, ça leur permet de se rendre compte qu'ils sont toujours des êtres humains, et qu'on les voit en tant qu'êtres humains et non pas en tant que détenus. (Personnel 7)

Quand on pense à la prison c'est toujours des formes qui sont carrées, qui sont dures, qui sont fortes, qui sont entrecroisées, tout est symétrique, géométrique (...) et là effectivement (avec les moulages exposés) on a affaire à des courbes, on a affaire à des formes, des rondeurs, des traits qui sont fins et délicats (...) on change le visage de la prison, et je pense que les détenus commencent à le ressentir, puisque je crois qu'ils ne sont pas étrangers à tout cela; ils savent très bien que c'est pour eux. (Personnel 4)

Apprendre des choses qu'on ne savait pas concernant une pratique artistique, découvrir un type de matériel, un vocabulaire, des techniques spécifiques, tout cet ensemble de connaissances nouvelles et d'apprentissages fait partie des bénéfices mentionnés par les détenus participant aux ateliers ainsi que par ceux participant aux conférences. Trois des détenus interrogés abordent la question de la réinsertion. Ils estiment que les ateliers les valorisent en ce sens qu'ils peuvent les aider à découvrir un métier ou à perfectionner leur connaissance de ce métier. Ainsi, les deux participants à l'atelier de

\footnotetext{
${ }^{55}$ Les verbatims cités sont extraits des entretiens avec les différents acteurs ayant accepté de témoigner de leur expérience. Ils sont anonymés.
} 
création sonore qui n'étaient pas opérateurs ${ }^{56}$ souhaitaient le devenir et ont fait une demande de formation au centre pénitentiaire de SaintMaur où la formation est organisée :

Ça peut être intéressant dans le sens où ça peut aider les détenus, surtout à la réinsertion, ça... Et puis former, former des artistes, former des gens quoi. Offrir une perspective. (Détenu participant 7)

Ici, on est encore jeunes, et je peux faire ce que je veux, ma passion, je peux encore devenir entraîneur de danse, ou entraîneur d'aïkido, ou encore champion de boxe. J'ai la capacité de le faire. (Détenu participant 10)

\section{L'espace de la démarche artistique}

Les trois ateliers menés à leur terme ont abouti à des productions. Le terme de "production » est ici utilisé pour qualifier, de manière générique, les objets qui ont été conçus puis fabriqués dans le cadre des ateliers. La relation tissée par les détenus avec ces productions est à l'origine de leur perception d'un autre espace de valeur qui est constitué de la démarche artistique qui consiste à ressentir des émotions fortes et intimes du fait de s'exprimer. Cette valeur consiste en un dépassement de soi :

(À travers ce type de projet, il s'agit de) donner la possibilité à ceux qui se sont toujours exprimés par la violence de s'exprimer enfin différemment. (Détenu non participant 2)

Je crois que c'est un droit qu'on peut prendre, celui de s'exprimer (...) Quand on s'exprime de façon artistique, on peut faire passer tous les messages qu'on veut. (Détenu non participant 2)

Il y a une chose qui est sûre et certaine, c'est que (le projet) va contribuer, que ça va contribuer à apaiser, à apaiser la colère qui est dans tout ce qu'on est ici, cette espèce d'univers très restreint, et si l'art peut avoir une mission ici, c'est celle-là. (Détenu participant 2)

Les personnes interrogées sont enthousiastes à l'idée que leurs productions puissent être diffusées en dehors de la prison, donc accessibles à leur famille notamment, mais aussi au grand public. La circulation des productions à l'extérieur de la prison a plus de valeur, pour les personnes détenues, que leur stricte diffusion au sein de la prison :

En tout cas, nous, si (l'intervenant de l'atelier de création sonore) nous demande s'il peut prendre les créations on lui dira oui. Ah ouais. Tout ce qui est artistique, c'est fait pour être diffusé donc... Et ça va pas être écouté par des milliers de gens. (Détenu participant 6)

En plus, ça va aller sur Internet, sur le site du Louvre, c'est excellent. (Détenu participant 3)

Néanmoins, dans le cadre de ce projet, les personnes détenues tardent à obtenir une trace matérielle - et donc symbolique - de leur production. Elles ne savent pas ce qu'il en adviendra et elles ne gardent pas non plus trace des statues enlevées à l'issue du projet. Elles se retrouvent, d'une certaine manière, " désœuvrés " une fois les ateliers et les conférences terminées et une fois les statues retirées de la cour 5. La valeur associée au dépassement de soi peut donc se révéler paradoxale. Elle met en tension le poids de la vie

\footnotetext{
${ }^{56}$ L'expression « opérateur » désigne les personnes détenues travaillant dans les ateliers de numérisation sonore, à la RIEP. Deux détenus parmi les participants de l'atelier de création sonore du projet des sculptures du Louvre n'étaient pas opérateurs.
} 
carcérale et la liberté de la création. Plusieurs détenus participants et non participants vont jusqu'à élaborer une analyse des risques que peut constituer le fait de terminer un projet et de laisser les personnes détenues aux prises avec leurs expériences de création :

\begin{abstract}
Mais sur des projets comme ça, je ne suis pas sûr que ce soit le regard de l'autre qui fasse peur, c'est surtout le regard de soi-même. [...] c'est plus la peur de l'échec. (Détenu non participant 5 participant Louvre 1)
\end{abstract}

Les détenus sont moins à l'abri que les autres, par rapport au syndrome de Stendhal, parce que, justement, on a tellement envie de s'évader que si l'occasion se présente, on s'évade comme ça. On sait très bien qu'on va revenir, mais plus on va loin, plus la souffrance est là au moment du retour. (Détenu non participant 2)

Les participants interrogés, pudiques sur l'attachement dont ils pourraient témoigner vis-à-vis de leurs productions, font tout de même état d'un lien avec ces productions. Ce lien s'exprime à travers une certaine anxiété vis-à-vis de la réception de ces productions lors de la journée de restitution :

Ici rien n'est gravé sur du marbre hein ! Le truc, c'est qu'une fois que la vidéo a quitté la prison et est au Louvre, alors la sécurité et l'Administration pénitentiaire ne peuvent plus rien faire. Moi, j'attends les nouvelles de l'intervenante de l'atelier vidéo, qu'elle me dise que tout est en ordre et corrigé et je serai complètement serein pour la restitution! (Détenu participant 1 )

\title{
L'émergence d'espaces de valeur collective
}

Le projet des sculptures engage aussi une autre forme de valeur qui n'a de sens que parce qu'elle est investie collectivement. Ses effets ont à voir avec le maintien ou l'existence d'un espace social ou d'un espace interpersonnel : (1) l'espace d'échanges et de relations entre personnes détenues et (2) l'espace de partage collectif.

L'espace d'échanges et de relations entre personnes détenues Les espaces de valeur collective sont le fruit des débats et des échanges entre personnes détenues, à l'occasion, d'une part, de l'introduction des sculptures dans la cour de la maison centrale et, d'autres part, des dynamiques collectives des ateliers de création. Cette valeur tient au fait que ces deux objets (les sculptures et les ateliers) ont ouvert un espace d'expression et d'échanges, dans un lieu qui ne favorise pas d'habitude les modalités d'échanges au sein de la population carcérale. Elle tient aussi au fait que, dans le premier cas, les deux moulages sont considérés par les détenus comme des objets dignes de valeur et que, dans le second cas, le travail en groupe est estimé bénéficier à tous les participants.

La présence des sculptures. Les copies des deux sculptures ont été installées en juillet 2012 dans la cour 5 de la maison centrale. Cette cour, on le rappelle, est essentiellement investie par les détenus, les surveillants et les autres personnels de la prison, comme un lieu de passage : elle permet l'accès à la RIEP, à I'UCSA ${ }^{57}$ et au bâtiment d'hébergement. Les deux sculptures peuvent donc être vues par l'ensemble de la population. Certains détenus, depuis leur cellule, ont « (la) vue » sur les deux sculptures, les autres passent devant en

\footnotetext{
${ }^{57}$ L'UCSA est l'Unité de Consultation et de Soin Ambulatoire, où sont assurés certains soins médicaux apportés aux personnes détenues.
} 
sortant de leur cellule et en y entrant. À ce titre, elles sont présentées à la vue de l'ensemble des détenus de la prison.

Dès l'arrivée des deux sculptures, leur emplacement - décidé par les paysagistes et les détenus participant à l'atelier horticulture - pose question aux détenus et aux personnels de surveillance. Les personnes interrogées ne savent pas pourquoi elles ont été placées à ces deux endroits-là :

Le choix des emplacements n'était pas judicieux. (...) C'est fou de mettre la Vénus Callipyge contre un mur (...) ils l'ont mise dans un coin qui ne correspond à rien. (Détenu participant 9 participant projet Louvre 1)

D'en avoir mis deux sur la cour 5 et rien du tout sur la cour 2, est-ce que c'est un choix judicieux ou pas? J'en sais rien. Je pense qu'on aurait dû équilibrer. (Personnel 4)

L'observation de la relation entre les détenus et les sculptures montre que les deux moulages font aussi l'objet de nombreux commentaires sur le sens de leur choix par le musée. Dans tous les cas, les deux sculptures apparaissent comme un objet de discours et de débats, et suscitent des échanges entre détenus et entre surveillants à propos de la religion et de ses interdits, de la nature artistique des sculptures ou encore des goûts de chacun :

\begin{abstract}
J'ai parlé avec des détenus, oui c'est vrai j'ai dit: "si je me lève tous les jours pour voir ça, sincèrement, c'est pas la peine". Pourquoi j'ai dit ça? Parce que je ne veux pas qu'ils en mettent un peu partout encore. Il y a ceux-là, ça suffit, si après tu bouges la tête et que tu ne vois que ça! (Détenu participant 10)

De toutes façons, toute discussion est intéressante, (...) il me disait : "ouais, ça me choque !", je lui ai dit: "mais dehors..." et c'est là qu'on voit qu'il y a un fossé et pas qu'avec la prison mais avec la société elle-même. "Quand t'étais dehors, tu traversais un square, un parc, t'as croisé des statues, ça t'a jamais gêné ?", il m'a dit: "j'en ai jamais vu !" (Détenu participant 9 participant projet Louvre 1)
\end{abstract}

Néanmoins, l'étude montre que l'appropriation des sculptures évolue avec le temps : si elles font l'objet d'un rejet à leur arrivée, elles font, petit à petit, l'objet d'une familiarité de la part des détenus qui regrettent, finalement, de les voir partir. L'appropriation s'est construite à travers plusieurs registres: les moulages créent un repère physique dans la prison ; ils créent un repère symbolique avec l'extérieur ; ils créent un repère temporel dans le cadre de la vie en prison :

Ça fait tellement longtemps qu'elles sont là... On s'approprie rapidement les choses en prison. Ça devient un repère, et un repère c'est rassurant. (Détenu non participant 2)

Elles vont laisser un grand vide ! (Détenu participant 7)

Les deux statues deviennent même un objet d'attachement. Elles n'ont pas été dégradées. Elles permettent, du point de vue de deux détenus interrogés, de regarder différemment un environnement qu'ils jugent dégradé. Elles convoquent des environnements connus à l'extérieur, des parcs et jardins ou des places publiques, et la référence à la statuaire de plein air :

Moi je trouve que ça change. Ça change, ça fait penser à un square, vous voyez? Ça a changé la cour complètement, parce que vous 
auriez vu l'état de la cour avant, c'était quelque chose d'autre... (Détenu participant conférence 1)

C'est bien aussi. Ca va... pas "embellir" la prison mais ça va lui apporter un plus quoi. C'est mieux d'avoir des fleurs, une pelouse, un endroit pour bouquiner, jouer aux échecs, que d'avoir un espace comme ça vide. (Détenu participant 7)

Les deux sculptures sont considérées, par les personnes interrogées, comme des objets de valeur, même par ceux qui précisent ne pas les apprécier. Le fait qu'il s'agisse de moulages n'a pas de répercussion sur la valeur que les enquêtés leur donnent : le moulage et l'original se confondent puisque la qualité de «moulage " n'est jamais évoquée. Ce qui compte, c'est la valeur du travail accompli aussi bien dans la production de l'objet, original ou copie, que dans son travail de restauration. On peut d'ailleurs noter que, sur les trois conférences observées $^{58}$, seul un des intervenants a parlé des sculptures exposées à Poissy, et il en a parlé en tant qu'œuvre et non en tant que moulage exposé dans la cour 5 . Les intervenants dont la conférence a fait l'objet d'une observation ne font donc pas non plus explicitement la distinction entre ces deux statuts :

Tu vois ce qui me fascine c'est, dans ces statues, la qualité des finitions alors qu'elles ont 2500 ans ! Ce travail... Comment c'est possible de faire une œuvre comme ça avec un petit marteau... (Détenu participant 1)

Même si je ne l'ai pas montré moi, j'ai conscience du travail que ça peut représenter... En amont comme en aval, quand elles (les statues) ont été faites pour de vrai et quand elles ont été reproduites par les petites mains du Louvre. (Détenu non participant 2)

Je ne sais pas ce que les gens ont compris sur la sculpture, qui était, en fin de compte, rapiécée, qu'on retrouve en entier, alors qu'en fin de compte elle était dans un état de délabrement, elle a été reconstituée, là on en a un modèle, c'est quelque chose d'extraordinaire mais je ne sais pas si les gens se rendent compte de la chance qu'ils ont d'avoir deux sculptures comme ça devant les yeux. (Détenu non participant 3)

La production collective des ateliers. Quel que soit l'atelier, les participants ont eu le sentiment d'être accompagnés dans leur pratique, surtout par les intervenants dans le cas de l'atelier de création sonore, et par les autres membres du groupe dans le cas de l'atelier vidéo et de l'atelier sculpture. Ces deux derniers ateliers ont abouti à des productions "collectives ", une vidéo et deux sculptures, que les participants estiment être le fruit d'un travail de groupe :

Les projets sont individuels d'un côté, mais c'est aussi un travail commun. Même si après je suis tout seul à un moment pour faire les derniers réglages, les derniers détails, on n'est quand même pas tout seul. D'un côté c'est un travail personnel mais on n'est pas abandonné. (Détenu participant 6)

D'abord on a fait des photos des statues, de leurs différentes faces, pour faire le montage. Le plus dur c'était ensuite d'ajuster les images entre elles et avec la musique. L'intervenante de l'atelier

\footnotetext{
58 Geneviève Bresc, directrice du Département des sculptures du Louvre, "Présentation de la Vénus Callipyge et des collections de sculptures du Louvre "; Georges Vigarello, directeur d'études à l'EHESS, « Le corps et le mouvement dans les arts visuels »; Daniel Ibled, restaurateur de sculptures et artiste, " La restauration des sculptures ». Une conférence par mois, parfois deux, ont été organisées entre septembre 2012 et mai 2013.
} 
vidéo passait l'image et faisait, avec la musique, clic, elle faisait "clic !" pour que ça tombe juste. À chaque fois qu'une image arrivait, elle coupait au rythme de la musique et $D$. le faisait aussi. (Détenu participant 1)

On a choisi l'idée de palmier (pour la sculpture) et après on s'est mis d'accord sur la brique, le Syporex (pour le matériau). (Détenu participant 7)

\section{Les espaces de partage}

Lors de la restitution, les créations sonores ont été diffusées les unes à la suite des autres avec, entre chaque création, l'intervention de deux musiciens jouant des pièces de quelques minutes composées par des compositeurs célèbres (Bartok, etc.) et par Nicolas Frize. L'ensemble, diffusé deux fois, durait quarante-cinq minutes à chaque fois. L'atelier vidéo a abouti à la création d'un film de quatre minutes, fruit d'un travail collectif entre les participants de l'atelier, diffusé plusieurs fois dans une salle du gymnase sur un écran. Enfin, l'atelier sculpture a permis de réaliser deux ouvrages: un banc et une sculpture, installés dans la cour 5.

Les participants ont été satisfaits de cette journée qu'ils ont estimée " ouverte à tous " et conviviale. Le fait d'organiser la restitution en deux parcours - un premier groupe de spectateurs visionne la vidéo pendant qu'un seconde groupe écoute les créations sonores ; puis le premier groupe écoute à son tour les créations sonores pendant que le second groupe visionne la vidéo - puis de réunir tous les participants (personnels de l'administration pénitentiaire, personnels du Louvre, personnels des administrations invitées, personnes détenues participantes et non participantes) autour de jus de fruits et de biscuits, a été très apprécié notamment en comparaison avec la restitution du projet précédent $A u$-delà des murs, du Louvre à la Maison centrale de Poissy, dont la première partie était réservée aux membres du projet et aux personnels de l'administration pénitentiaire :

Cette journée-là, elle s'est bien passée ; c'était une bonne journée et on nous la prendra plus. Ça nous fait un beau souvenir, ça c'est fait, c'était bien, et quelque part ça reste là (présent dans la mémoire). (Détenu participant 6)

A priori, euh, la façon dont ça a été présenté, euh, le fait qu'il n'y ait pas de journaliste, le fait que ce ne soit pas un truc "champagne", ça a préservé ce côté-là quoi. C'est vachement important, quoi, pour que les gens puissent s'exprimer pleinement. Non, ça a été très bien fait. (Détenu participant 2)

Les créations sonores et le film ne sont destinés à être diffusés à la maison centrale que lors de la journée de restitution. La situation est différente pour les deux productions de l'atelier sculpture dans la mesure où elles ont été installées de façon pérenne dans la cour 5. Les créations sonores ont été appréciées, d'autant plus que le dispositif scénique permettait une situation d'écoute attentive (les assises, la présentation par Nicolas Frize, les interludes musicaux avec les deux musiciens). Les participants ont été interpellés par d'autres détenus qui ont salué le travail accompli. En revanche, concernant la production vidéo, les témoignages sont peu nombreux, mais la création semble avoir été appréciée par les détenus qui en ont été spectateurs :

Les mecs ils m'ont dit: "c'est vraiment bien", (...) ils ont trouvé que c'était plutôt pas mal. Ouais et puis, (ils ont été) surpris je pense, 
moi, plus qu'impressionnés, de se dire: "ah tiens quand même, ils font des trucs, ils savent faire ça eux." (Détenu participant 6)

Mais je ne pensais pas que ce serait aussi sympa, avec les petits coussins, l'organisation comme elle était faite avec les groupes à dimension convenable, les groupes qui se croisent (silence) des petits ateliers... C'était sympa ouais. Une bonne après-midi ouais. (Détenu participant 2)

\section{Conclusion}

En suscitant des situations d'interaction entre les participants issus de l'extérieur ou de la maison centrale durant plusieurs mois, en rendant possible un acte de création, en rendant visible la présence du Louvre et de l'art par l'exposition des deux moulages de sculpture, le projet a bien permis de faire émerger un sentiment commun de la valeur de ce qui a été produit collectivement dans les ateliers, mais aussi de ce qui a été collectivement mis en valeur à travers le respect et la considération des moulages et lors de la restitution des créations face à un public, créant ainsi des espaces d'intérêt général, certes fugaces et rendus possibles, semble-t-il, parce qu'ils rappelaient l'existence du monde extérieur. Enfin, à titre individuel, se sentir autre que détenu, et faire les choses dans son propre intérêt, n'était sans doute pas possible pour tous, néanmoins, le projet du Louvre, en créant les conditions d'échanges désintéressés et d'expression de soi, a permis à certains de se reconnaître à eux-mêmes une certaine valeur.

\section{Références}

Andrieu, M. (2011). «Réalités musicales en prison: D’un panorama général à l'analyse d'une activité. » Dans Benguigui, G. \& Guilbaud, F. \& Malochet, G. (Ed.). La prison sous tension. (pp. 306-335). Nîmes : Champ social.

Becker, H. (1988). Les Mondes de l'art. Paris : Flammarion.

Benguigui, G. (2011). "La paranoïa pénitentiaire. » Dans G. Benguigui \& F. Guilbaud \& G. Malochet (Ed.). La prison sous tension. (pp. 57-87). Nîmes : Champ social.

Chauvenet, A., Rostaing, C., \& Orlic, F. (Ed.). (2008). La Violence carcérale en question. Paris : Presses Universitaires de France.

Chauvenet, A. (2006). "Privation de liberté et violence: Le despotisme ordinaire en prison. » Déviance et société, 30, 3, (pp. 373-388).

Frize, N. (2004). Le Sens de la peine. Paris : Lignes / Léo Scheer.

Jeanneret, Y. (2011). Where is Mona Lisa? Et autres lieux de la culture. Paris : Le Cavalier bleu.

Lehmann, J.-P. (2009). Comprendre Winnicott. Paris : Armand Colin.

Musée du Louvre-Service Etudes et Recherche (2010). Évaluer un dispositif artistique et culturel en milieu pénitentiaire. Un partenariat entre le musée du Louvre, le Service pénitentiaire d'insertion et de probation de Paris et la Maison d'arrêt de la Santé, rapport d'enquête qualitative. [Non publié]

Musée du Louvre-Service Etudes et Recherche (2013). Évaluer un dispositif artistique et culturel en milieu pénitentiaire. Au-delà des murs, du Louvre à la Maison centrale de Poissy, rapport d'enquête qualitative. [Non publié]

Rostaing, C., \& Touraut, C. (2012). « Processus de création culturelle en prison: Une innovation ordinaire?" Socio-logos: Revue de l'association française de sociologie, 7. Consulté à partir de http://socio-logos.revues.org/2658.

Salle, G. (2011). « 1975 : Une date marquante dans l'histoire de la prison? Petit essai de mise en perspective. » Dans Benguigui, G. \& 
Guilbaud, F. \& Malochet, G. (Ed.) La prison sous tension. (pp. 2056). Nîmes : Champ social.

Siganos, F. (2008). L'Action culturelle en prison. Paris : Ed. de l'Harmattan. Soulier, G. (1985). La Culture en prison, quels enjeux ? Actes du colloque de Reims. Paris : La Documentation française.

Winnicott, D. W., (1975 [1971]). Jeu et Réalité : L'espace potentiel. Paris : Gallimard. 


\section{Résumé}

Cet article analyse les effets des projets culturels en contexte pénitentiaire. II s'appuie sur les résultats d'une enquête par entretiens et observations auprès des acteurs du «projet des sculptures " mené par le musée du Louvre à la maison centrale de Poissy entre septembre 2012 et mai 2013. Ce projet s'articulait autour de l'installation de deux moulages de sculptures du musée et de la programmation de quatre ateliers de création ainsi que d'un cycle de conférences. Cet article interroge le répertoire de valeurs qui peut émerger de ces activités selon les personnes détenues. L'analyse des résultats de l'enquête montre que ce répertoire se déploie à l'échelle individuelle mais aussi, différemment, à l'échelle collective. Les espaces de valeur individuelle sont ceux de la relation avec les intervenants et ceux de la démarche artistique et créative. Les espaces de valeur collective sont le fruit de débats et d'échanges entre personnes détenues à l'occasion, d'une part, de l'introduction des sculptures dans la maison centrale et, d'autre part, des dynamiques collectives des ateliers de création.

Mots clé : projets culturels, prisons, étude du public, Musée du Louvre

\section{Abstract}

Creating value in a penitentiary context: the artistic and cultural project of the Louvre Museum in the penitentiary at Poissy

This article analyses the effects of cultural projects in the context of a prison. It examines the results of interviews and observations from detainees participating in the "sculpture project" led by the Louvre in the Poissy between September 2012 and May 2013. This project was built around the installation of two casts of sculptures from the museum, four creative workshops, along with a series of lectures. This article examines the value that these activities brought according to the detainees. Interviews and observations show that these events were felt at both the individual and the collective level, although differently. Individual value was experienced throughout the relationship with the artists in charge of the workshops, and in the process of the artistic and creative workshop. Collective value was the result of discussions and exchanges among the detainees due to, on the one hand, the introduction of sculptures into the prison, and, on the other hand, the collective dynamics of the workshops.

Key words: cultural projects, prisons, visitor studies, Louvre Museum

\section{Resumen}

Creación de Valor en Contexto de una Penitenciaría : El Proyecto Artístico y Cultural del Museo del Louvre en la Casa Central de Poissy

En este artículo se analizan los efectos de los proyectos culturales en el contexto penitenciario. Se basa en los resultados de una encuesta (entrevistas y observaciones) con los participantes al "proyecto de las esculturas", llevado a cabo por el Louvre en la cárcel de Poissy, entre septiembre de 2012 y mayo de 2013. Este proyecto se estructura en torno a la instalación de dos moldes de esculturas del museo y la programación de cuatro talleres creativos y una serie de conferencias. Este artículo examina el repertorio de valores que pueden surgir de estas actividades de acuerdo con los detenidos. Análisis de los resultados de la encuesta muestran que este repertorio se despliega a nivel individual, y también a nivel colectivo. Los espacios de valor individual son los de la relación con los responsables de los talleres, y los de la actividad artística y creativa. Los espacios de valor colectivo son el resultado de las discusiones y los debates entre las personas detenidas a raíz de la introducción de las esculturas en la cárcel y, por otro lado, de las 
dinámicas colectivas en los talleres.

Palabras clave: proyecto cultural, penitenciaría, publico, Museo del Louvre 